\title{
Effect of Partially Coherent Light on the Contrast of Speckle Patterns Obtained Using Digital Image Processing of Speckle Photography
}

\author{
Nasser A. Moustafa, Mohamad M. El-Nicklawy, Amin F. Hassan, Amany K. Ibrahim \\ Department of Physics, Faculty of Science, Helwan University, Cairo, Egypt \\ Email: Nasseramoustafa@yahoo.com
}

Received May 13, 2013; revised June 17, 2013; accepted July 12, 2013

Copyright (C) 2013 Nasser A. Moustafa et al. This is an open access article distributed under the Creative Commons Attribution License, which permits unrestricted use, distribution, and reproduction in any medium, provided the original work is properly cited.

\begin{abstract}
The paper is devoted to study theoretically, the effects of some parameters on the visibility of the speckle patterns. For this propose, a theoretical model for a periodic rough surface was considered. Using this theoretical model, the effects of grain height, its density, the band width and spectral distribution of the line profile (Gaussian and Lorentzian) illuminating a rough surface on the visibility of speckle pattern are investigated. An experimental setup was constructed to study the effect of surface roughness and coherence of the illuminating light beam on the contrast of speckle pattern. The general behavior of the experimental results, which agree with published data, is compatible with the new theoretical model.
\end{abstract}

Keywords: Speckle Pattern; Surface Roughness; Partially Coherent Light

\section{Introduction}

Speckle metrology techniques are promise to be a fruitful approach for solving a number of difficult problems in industry. The speckle pattern technique seems to be well suited for problems involving surface phenomena, fragile specimens and others [1-3]. A speckle pattern formed by partially spatially coherent light has been studied theoretically by many authors [4-6]. Fujii and Asakura [5] have developed a theoretical formulae for the intensity distribution of the speckle pattern as a function of the temporal coherence function and an equivalent optical transfer function. The relation between the statistical properties of the speckle pattern and the coherence characteristics of the illuminating source have been studied by Asakura et al. [7], Fujiwara et al. [8] and Parry [9]. El-Nicklawy et al. [10] is devoted to the study of surface roughness from an interferometric point of view. It deals with a theoretical investigation of the intensity and visibility distribution of the speckle pattern resulting from the transmitted scattered radiation of strictly- and quasi monochromatic beams with Gaussian, Lorentzian and Voigte spectral profiles. The present work studied the effect of partially coherent light on the visibility of the speckle pattern. A theoretical model for a periodic rough surface was constructed. An equation for the visibility of speckle patterns is investigated by using partially coherent light.

\section{Theoretical Model}

Suppose a grain of dimension $(a, b)$ is located on the surface of the transparent diffuser (Figure 1), where its center having the coordinates $\left(x_{i}, y_{i}, 0\right)$. The wave function scattered from an element of dimensions $\left(d x_{i}, d y_{i}, 0\right)$ located at position $(x, y, 0)$ and illuminating a point $P$ of coordinates $(X, Y, Z)$ on a screen placed a distance apart from the diffuser is given by

$$
\Psi=\frac{a_{0}}{r} \mathrm{e}^{i(w t-k r)} \mathrm{d} x \mathrm{~d} y
$$

where $a_{0} / r$ is the scattered amplitude per unit area from the diffuser,

$$
r^{2}=(X-x)^{2}+(Y-y)^{2}+Z^{2}
$$

Let the grain under study is of dimensions $a, b$, and position $\left(x_{i}, y_{i}, 0\right)$.

$$
R^{2}=\left(X-x_{i}\right)^{2}+\left(Y-y_{i}\right)^{2}+Z^{2}
$$

For $X \gg x$ and $x_{i}$, and $Y \gg y$ and $y_{i}$ we get:

$$
r=R\left[1-\frac{2 X\left(x-x_{i}\right)}{R^{2}}-\frac{2 Y\left(y-y_{i}\right)}{R^{2}}\right]^{1 / 2}
$$




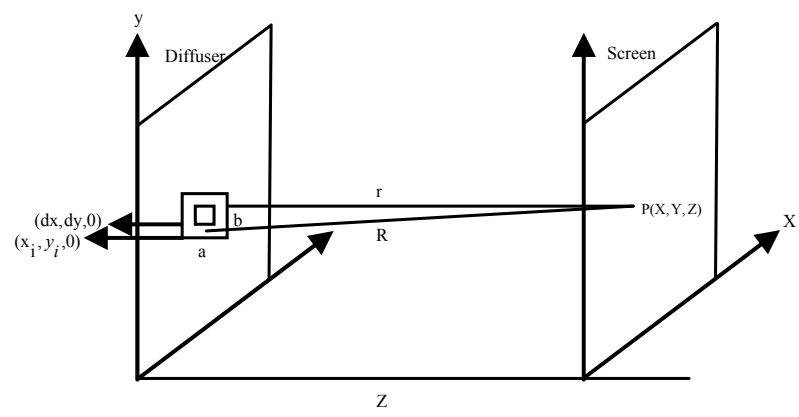

Figure 1. Construction of the theoretical model.

Using the Bernwlli inequality, one gets

$(1+\xi)^{n} \approx 1+\frac{n \xi}{1 !}$ for $\xi \ll 1$. Thus $r$ can be written as

$$
r \approx R-\frac{X\left(x-x_{i}\right)}{R}-\frac{Y\left(y-y_{i}\right)}{R}
$$

The integration of the wave amplitude given by Equation (5), over the entire area of a grain pensioned on $\left(x_{i}\right.$, $\left.y_{i}, 0\right)$ gives the resultant wave amplitude emitted from and reaching point $P$ on the screen as

$$
\begin{gathered}
A_{g 1} \mathrm{e}^{i \phi_{i}}=\int_{x_{i}-\frac{a}{2}}^{x_{1}+\frac{a}{2}} \int_{y_{i}-\frac{b}{2}}^{y_{i}+\frac{b}{2}} \mathrm{e}^{\frac{a_{0}}{r}} \mathrm{e}^{i(w t-k r)} \mathrm{d} x \mathrm{~d} y \\
A_{g 1} \mathrm{e}^{i \phi_{i}}=\frac{a_{0}}{R} \mathrm{e}^{i(w t-k R)}\left[\frac{\mathrm{e}^{i \frac{k X\left(x-x_{i}\right)}{R}}}{i \frac{k X}{R}}\right]_{x_{i}-\frac{a_{0}}{2}}^{x_{i}+\frac{a_{0}}{2}}\left[\frac{\mathrm{e}^{i \frac{k Y\left(y-y_{i}\right)}{R}}}{i \frac{k Y}{R}}\right]_{y_{i}-\frac{a_{0}}{2}}^{y_{i}+\frac{a_{0}}{2}} \\
A_{g 1} \mathrm{e}^{i \phi_{i}}=\frac{a_{0} a b}{R} \mathrm{e}^{i(w t-k R)}\left[\frac{\sin \left(\frac{k X a}{2 R}\right)}{\frac{k X a}{2 R}}\right]\left[\frac{\sin \left(\frac{k Y b}{2 R}\right)}{\frac{k Y b}{2 R}}\right]
\end{gathered}
$$

Let now another type of grain of thickness

$$
\delta \phi=k \delta R(\mu-1)=\frac{k Z \delta z(\mu-1)}{R}
$$

where $\mu$ is the refracted index of used diffuser. Since $R \ll \delta R$ the amplitude is considered to be constant. Thus the wave amplitude reaching the point $P$ from the second grain type will be given by:

$$
A_{g 2} \mathrm{e}^{i \phi_{i}}=\frac{a_{0} a b}{R} \mathrm{e}^{i(w t-k R-\delta \phi)}\left[\frac{\sin \left(\frac{k X a}{2 R}\right)}{\frac{k X a}{2 R}}\right]\left[\frac{\sin \left(\frac{k X b}{2 R}\right)}{\frac{k X b}{2 R}}\right]
$$

with

$$
R^{2}=X^{2}+Y^{2}+Z^{2}-2 X x_{i}-2 Y y_{i}=R_{0}^{2}\left[1-\frac{2 X x_{i}}{R_{0}^{2}}-\frac{Y y_{i}}{R_{o}^{2}}\right]
$$

where $R_{0}^{2}=X^{2}+Y^{2}+Z^{2}$. Using the binomial expansion theorem $(1+x)^{n}=1+\frac{n x}{1 !} \frac{n(n-1)}{2 !} x^{2}+\cdots$ one gets after excluding the higher terms $R \approx R_{0}-\frac{X x_{i}}{R_{0}}-\frac{Y y_{i}}{R_{0}}$.

Let now an $N \times N$ number of grains of the first type per unit area be resident on the diffuser, thus the resultant wave amplitude reaching the point $P$ on the screen will be given by:

$$
\begin{aligned}
& A_{g 1} \mathrm{e}^{i \phi_{i}}=\frac{a_{0} a b}{R} \mathrm{e}^{i\left(w t-k R_{0}\right)}\left[\frac{\sin \left(\frac{k X a}{2 R}\right)}{\frac{k X a}{2 R}}\right]\left[\frac{\sin \left(\frac{k X b}{2 R}\right)}{\frac{k X b}{2 R}}\right] \\
& \mathrm{e}^{i \frac{k}{R_{0}} \frac{a}{2} X} \sum_{l=1}^{N} \mathrm{e}^{i \frac{k}{R_{0}} 2 a(l-1) X} x \mathrm{e}^{i \frac{k}{R_{0}} \frac{b}{2} Y} \sum_{l=1}^{N} \mathrm{e}^{i \frac{k}{R_{0}} 2 b(l-1) Y}
\end{aligned}
$$

i.e.

$$
\begin{aligned}
& A_{g 1} \mathrm{e}^{i \phi_{i}}=\frac{a_{0} a b}{R} \mathrm{e}^{i\left(w t-k R_{0}\right)}\left[\frac{\sin \left(\frac{k X a}{2 R}\right)}{\frac{k X a}{2 R}}\right]\left[\frac{\sin \left(\frac{k X b}{2 R}\right)}{\frac{k X b}{2 R}}\right] \\
& x \mathrm{e}^{i \frac{k}{R_{0}}\left(\frac{a X+b Y}{2}\right)} x \frac{1-\mathrm{e}^{i N \delta_{1}}}{1-\mathrm{e}^{i \delta_{1}}} \cdot \frac{1-\mathrm{e}^{i N \delta_{2}}}{1-\mathrm{e}^{i \delta_{2}}}
\end{aligned}
$$

where $\delta_{1}=\frac{k}{R_{0}}(2 a) X$, and $\delta_{2}=\frac{k}{R_{0}}(2 b) Y$.

Similarly for the second type of the grains of number $N \times N$ per unit area, the resultant wave reaching the point $P$ will be given by:

$$
\begin{aligned}
& A_{g 2} \mathrm{e}^{i \phi_{2}}=\frac{a_{0} a b}{R} \mathrm{e}^{i\left(w t-k R_{0}\right)}\left[\frac{\sin \left(\frac{k X a}{2 R}\right)}{\frac{k X a}{2 R}}\right]\left[\frac{\sin \left(\frac{k X b}{2 R}\right)}{\frac{k X b}{2 R}}\right] \\
& x \mathrm{e}^{i \delta \phi} \cdot \mathrm{e}^{i \frac{k}{R_{0}}\left(\frac{3(a X+b Y)}{2}\right)} x \frac{1-\mathrm{e}^{i N \delta_{1}}}{1-\mathrm{e}^{i \delta_{1}}} \cdot \frac{1-\mathrm{e}^{i N \delta_{2}}}{1-\mathrm{e}^{i \delta_{2}}}
\end{aligned}
$$

From Equations (11) and (12), it is seen that the phase difference between the two resultant waves $\left(\phi_{2}-\phi_{1}\right)$ is given by:

$$
\begin{aligned}
\left(\phi_{2}-\phi_{1}\right) & =\delta \phi+\frac{k}{R_{0}}(a X+b Y) \\
& =\frac{k Z \delta z(\mu-1)}{R}+\frac{k}{R_{0}}(a X+b Y)
\end{aligned}
$$

Since

$$
I=\left|A_{g 1}\right|^{2}+\left|A_{g 2}\right|^{2}+2\left|A_{g 1}\right|\left|A_{g 2}\right| \cos \left(\phi_{2}-\phi_{1}\right)
$$

with $\left|A_{g 1}\right|=\left|A_{g 2}\right|=|A| \quad$ Equation (14) becomes 


$$
I=2|A|^{2}\left[1+\cos \left(\phi_{2}-\phi_{1}\right)\right]
$$

For $\frac{X x_{i}}{R_{0}}$ and $\frac{Y y_{i}}{R_{0}} \ll R_{0}$, it follows $R \approx R_{0}$ and $\left(\phi_{2}-\phi_{1}\right)=\frac{k Z \delta z(\mu-1)}{R_{0}}+\frac{k}{R_{0}}(a X+b Y)$.

Setting $Y=0$, and $\sin \left(\frac{\delta_{1}}{4}\right) \approx \sin \left(\frac{\delta_{1}}{2}\right)|A|^{2}$ can be rewrite in the form

$$
|A|^{2}=\left(\frac{a_{0} a b}{R}\right)^{2}\left[\frac{\sin \left(\frac{N \delta_{1}}{2}\right)}{\frac{\delta_{1}}{4}}\right]^{2}
$$

with $\sin (\theta)=\theta-\frac{\theta^{3}}{3 !}+\frac{\theta^{5}}{5 !}-\frac{\theta^{7}}{7 !}+\cdots$,

$$
\cos (\theta)=1-\frac{\theta^{2}}{2 !}+\frac{\theta^{4}}{4 !}-\frac{\theta^{6}}{6 !}+\cdots
$$

and

$$
\begin{gathered}
\cos \left(\frac{N \delta_{1}}{2}\right) \approx 1-\frac{\left(N \delta_{1} / 2\right)^{2}}{3 !}+\frac{\left(N \delta_{1} / 2\right)^{4}}{5 !}+\cdots \\
\therefore|A|^{2} \approx\left(\frac{a_{0} a b}{R}\right)^{2} 4 N^{2}\left(\cos \left(\frac{N \delta_{1}}{2}\right)\right)^{2}
\end{gathered}
$$

Thus the intensity can be written as

$$
\therefore I=2\left(\frac{a_{0} a b}{R}\right)^{2} 4 N^{2}\left(\cos \left(\frac{N \delta_{1}}{2}\right)\right)^{2}\left[1+\cos \left(\phi_{2}-\phi_{1}\right)\right]
$$

Setting $\phi_{2}-\phi_{1}=m v$, where

$$
\begin{gathered}
m=\left(\frac{2 \pi}{c}\right)\left[\frac{((Z \delta z(\mu-1))+(a X))}{R}\right] \\
\text { and } \delta_{1}=g v, \text { where } g=\left(\frac{2 \pi}{c}\right)\left[\frac{2 a X}{R}\right] \\
\therefore I=8\left(\frac{a_{0} a b}{R}\right)^{2} N^{2}\left(\cos \left(\frac{N g v}{2}\right)\right)^{2}[1+\cos (m v)]
\end{gathered}
$$

In the forgoing derivation, monochromatic irradiation of the rough surface was considered. In the paragraph to follow, the rough surface will be assumed to be non-monochromatic having different spectral distribution.

\subsection{Gaussian Spectral Distribution}

Assuming a symmetrical spectral line profile around $v_{0}$ having a half width $\Delta v$ and given by the

$$
g(v)=\left|a_{0}(v)\right|^{2}=\left|a_{g}(v)\right|^{2} \mathrm{e}^{-\alpha\left(v-v_{0}\right)^{2}}
$$

to irradiate the rough surface of the target where $\left|a_{g}(v)\right|^{2}=\sqrt{\frac{\alpha}{\pi}}$ and $\alpha=\frac{4 \ln 2}{(\Delta v)^{2}}$.

The intensity on the screen might be given by:

$$
\begin{gathered}
I_{G}=\int_{-\infty}^{\infty} I g(v) \mathrm{d} v \\
I_{G}=\int_{-\infty}^{\infty} 8\left(\frac{a_{0} a b}{R}\right)^{2} N^{2}\left(\cos \left(\frac{N g v}{2}\right)\right)^{2}[1+\cos (m v)] \\
\left|a_{g}(v)\right|^{2} \mathrm{e}^{-\alpha\left(v-v_{0}\right)^{2}} \mathrm{~d} v
\end{gathered}
$$

Setting

$$
\begin{aligned}
& c_{G}=4\left|a_{g}(v)\right|^{2}\left(\frac{a_{0} a b}{R}\right)^{2} N^{2}, \\
& \left(\cos \left(\frac{N g v}{2}\right)\right)^{2}=\frac{1}{2}[1+\cos (N g v)], \quad v-v_{0}=x \text { and } \\
& \mathrm{d} v=\mathrm{d} x \\
& I_{G}=C_{G} \int_{-\infty}^{\infty}\left[1+\cos \left(N g\left(x+v_{0}\right)\right)+\cos \left(m\left(x+v_{0}\right)\right)\right. \\
& \left.+\cos \left(N g\left(x+v_{0}\right)\right) \cos \left(m\left(x+v_{0}\right)\right)\right] \mathrm{e}^{-\alpha x^{2}} \mathrm{~d} x
\end{aligned}
$$

The integration of (26) gives, with $N_{g} \ll m$ the following expression for the intensity distribution

$$
I_{G}=C_{G} \sqrt{\frac{\pi}{\alpha}}\left(1+2 \cos \left(m v_{0}\right) \mathrm{e}^{-\frac{m^{2}}{4 \alpha}}+\cos \left(N_{g} v_{0}\right) \mathrm{e} \frac{-\left(N_{g}\right)^{2}}{4 \alpha}\right)
$$

From (27) it is obvious that the intensity reaches its maximum as $N_{g} \nu_{o}=2 m \pi$, and its minimum as $N_{g} \nu_{o}=(2 m+1) \pi$, with $m=0,1,2,3, \cdots$. Considering the above conditions for maximum and minimum intensity in (27) and setting the obtained expressions for $I_{G \text { max. }}$ and $I_{G \text { min. }}$, one obtains,

$$
V_{G}=\frac{\mathrm{e} \frac{-\left(N_{g}\right)^{2}}{4 \alpha}}{\left[1+\left(2 \cos \left(m v_{0}\right) \mathrm{e}^{-\frac{m^{2}}{4 \alpha}}\right)\right]}
$$

\subsection{Lorentzian Spectral Distribution}

In this case it is assumed that a symmetrical spectral line profile around $v_{o}$ following a Lorentizian profile function,

$$
\left|a_{0}(v)\right|^{2}=\left|a_{L}\left(v_{0}\right)^{2}\right| \frac{1}{1+\left(\frac{v-v_{0}}{\Delta v / 2}\right)^{2}}
$$


where $\left|a_{L}\left(v_{0}\right)^{2}\right|=\frac{2}{\pi \Delta v}$

The intensity distribution is

$$
\begin{gathered}
I_{L}=\int_{-\infty}^{\infty} I\left|a_{o}\left(v_{0}\right)\right|^{2} \mathrm{~d} v \\
{\left[1+\cos \left(N g\left(x+v_{0}\right)\right)\right]} \\
I_{L}=C_{L} \int_{-\infty}^{\infty}\left[1+\cos \left(m\left(x+v_{0}\right)\right)\right] \frac{1}{1+\left(\frac{x}{\Delta v / 2}\right)^{2}} \mathrm{~d} x
\end{gathered}
$$

where $C_{L}=4\left|a_{L}(v)\right|^{2} \frac{a_{0} a b}{R} N^{2}$

For $N_{g} \ll m$,

Then $I_{L}$ can be written as

$$
I_{L}=C_{L} \frac{\pi \Delta v}{2}\left[1+2 \cos \left(m v_{0}\right) \mathrm{e}^{-\frac{m \Delta v}{2}}+\cos \left(N_{g} v_{0}\right) \mathrm{e}^{\frac{-N_{g} \Delta v}{2}}\right]
$$

From (32) it is obvious that the intensity is a maximum as $N_{g} \nu_{o}=2 m \pi$, and its minimum as $N_{g} \nu_{o}=(2 m+1) \pi$, with $m=0,1,2,3, \cdots$. Setting the above conditions in (32), one gets after determining $I_{L \max }$ and $I_{L \min }$. the following equation for the visibility

$$
V_{L}=\frac{\mathrm{e}^{\frac{-N_{g} \Delta v}{2}}}{\left[1+\left(2 \cos \left(m v_{0}\right) \mathrm{e}^{-\frac{m \Delta v}{2}}\right)\right]} .
$$

\section{Results and Discussion}

To illustrate the dependence of the visibility on the grain height (surface roughness, the spectral half width, and the density of the grains, Equations (28) and (33) are computed for great distance between the diffuser and the screen, this distance chosen in the computation to be equal $400 \mathrm{~cm}$. The area of the diffuser is constant $=1$ $\mathrm{cm}^{2}$. Figure 2 shows the obtained results between the visibility of the speckle patterns and the spectral half width. The calculation was carried out considering the grain height and height to be $10^{6}$ in $\mathrm{cm}^{2}$ and $50 \mu \mathrm{m}$ respectively. From the figure it is evident that the visibility of Gaussian distribution is greater than that obtained from Lorentzian one considers the same half width is due to the higher effective spectral band width in the Lorentzian distribution which is larger than that in the case of the Gaussian one. The obtained behavior is in agreement with experimental data given in $[6,11,12]$.

At a grain height of $10 \mu \mathrm{m}$, a spectral half width of $10^{12} \mathrm{~Hz}$ and varying grain width, Equations (28) and (33) were computed to study the effect of the grain density on the visibility of speckle patterns. Figure 3 shows the

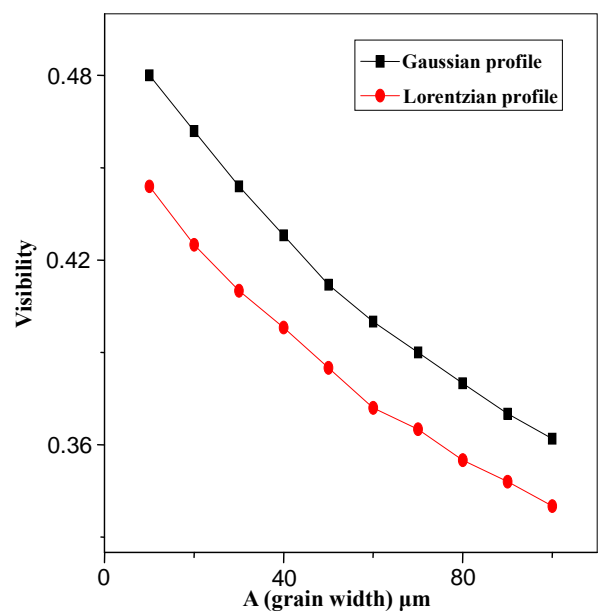

Figure 2. The visibility of speckle patterns versus the spectral half width $\Delta v$ with spectral line shape as a parameter. The results are obtained considering the grain density to be $10^{6}$ in $\mathrm{cm}^{2}$, the grain height to be $50 \mu \mathrm{m}$.

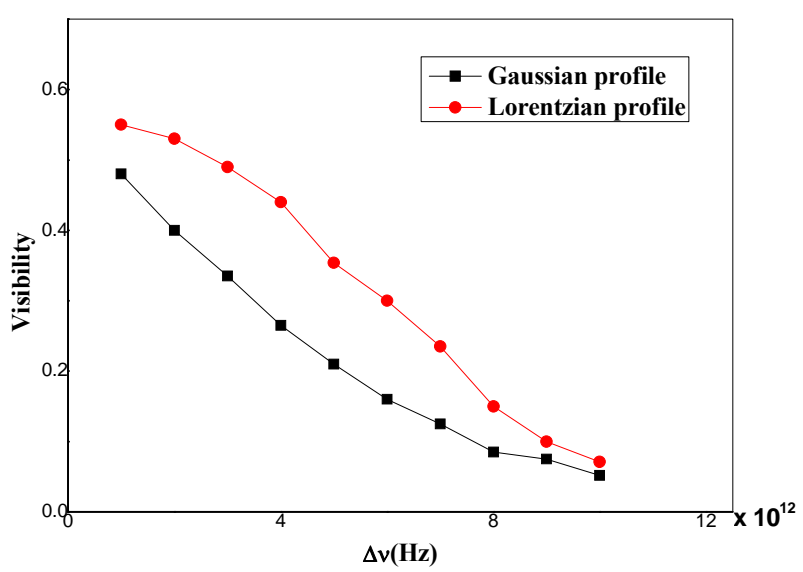

Figure 3. The visibility of the speckle patterns versus the grain width for the case of the Gaussian and Lorentzian profiles. The results are obtained for a grain height of $\mathbf{1 0}$ $\mu \mathrm{m}$ and spectral half width of $10^{12} \mathrm{~Hz}$.

dependence of the visibility of speckle patterns on the grain width in case of Gaussian and Lorentzian profiles. It is evident that the greater density of the grains yields higher visibility of the speckle patterns. This behavior can be interpreted by the following: By increasing the density, the randomization in difference between the interfering beam become smaller leading to increase the visibility.

By keeping grain width equals $10 \mu \mathrm{m}$ and varying the grain height, Equations (28) and (33) were computed also to study the effect height on the visibility of the speckle patterns. Figure 4 shows the dependence of the visibility of speckle patterns on the grain height in case of Gaussian and Lorentzian spectral distributions at $\Delta v=10^{12} \mathrm{~Hz}$. Figure 5 shows also the same, but for the case of Gaussian and Lorentzian spectral distributions at $\Delta v=10^{13} \mathrm{~Hz}$. It is evident that by increasing the half width of the ra- 


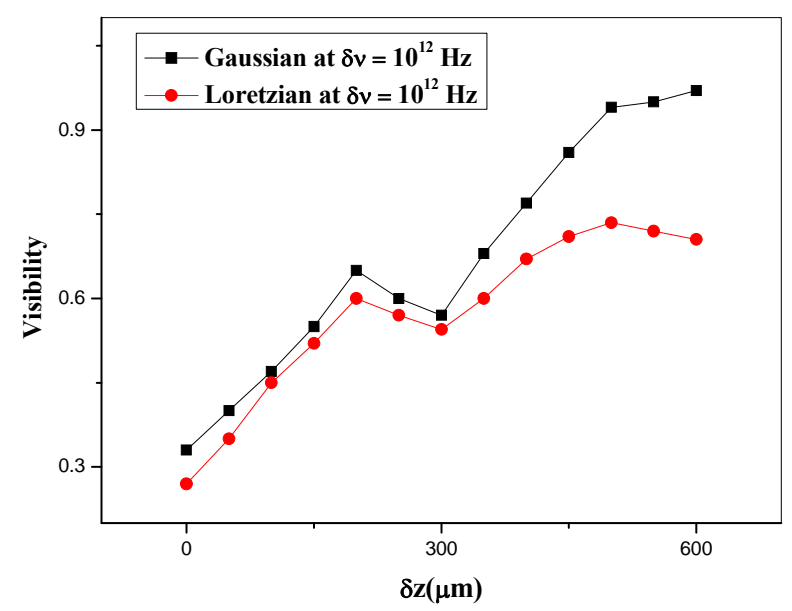

Figure 4. The visibility of the speckle patterns versus the grain height calculated for the case of Gaussian and Lorentzian profiles of $\delta v=10^{12} \mathrm{~Hz}$. The results are obtained for a grain density of $10^{6}$ in $\mathrm{cm}^{2}$.

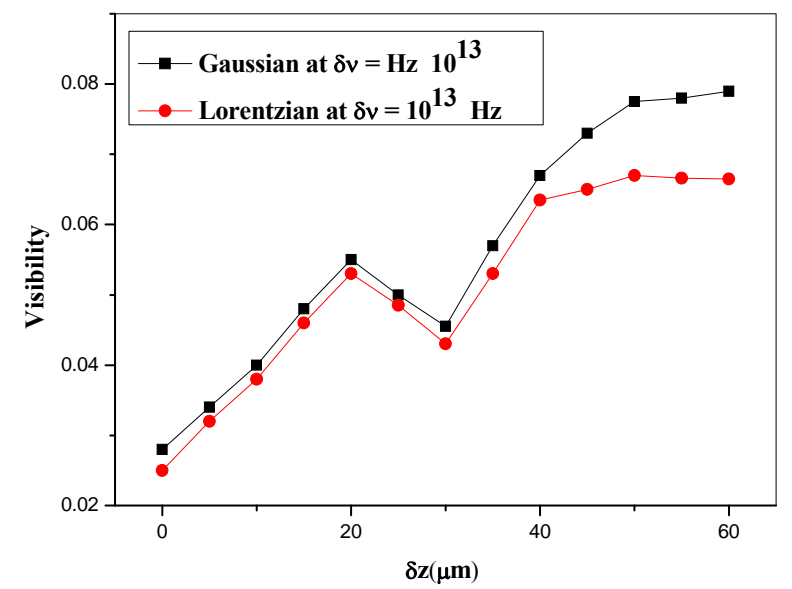

Figure 5. The visibility of the speckle patterns versus the grain height calculated for the case of Gaussian and Lorentzian profiles of $\delta v=10^{13} \mathrm{~Hz}$. The results are obtained for a grain density of $10^{6}$ in $\mathrm{cm}^{2}$.

diation, the visibility of speckle patterns decreases. It is due to the inverse dependence of the degree of coherence of the light beam and its half width.

\section{Experimental Verification}

To verify the theoretical model an experiment as shown in Figure 6 was carried out using a partially coherent light obtained from a mercury lamp. The collimated light illuminates a diffusely transmitting object having different surface roughnesses. The obtained speckle intensity distributions were captured by a webcam (digital camera). To find the relation between the speckle intensity fluctuation and the surface roughness, the surface roughnesses of these objects was precisely mechanically measured beforehand by suing a stylus instrument. Figure 7 shows the obtained speckle pattern from partially

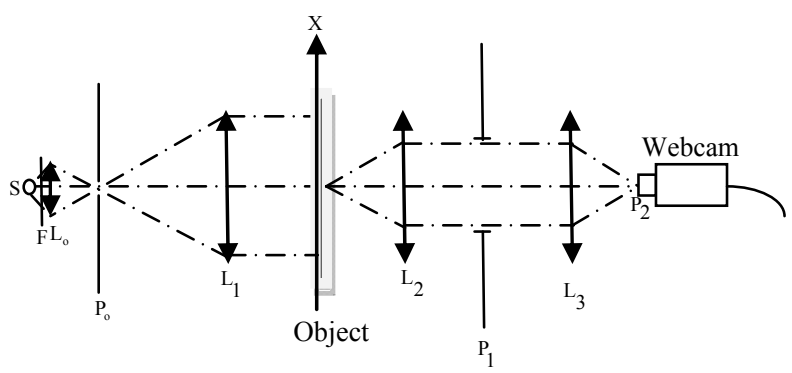

Figure 6. The experimental setup used for the verification of the theoretical model.

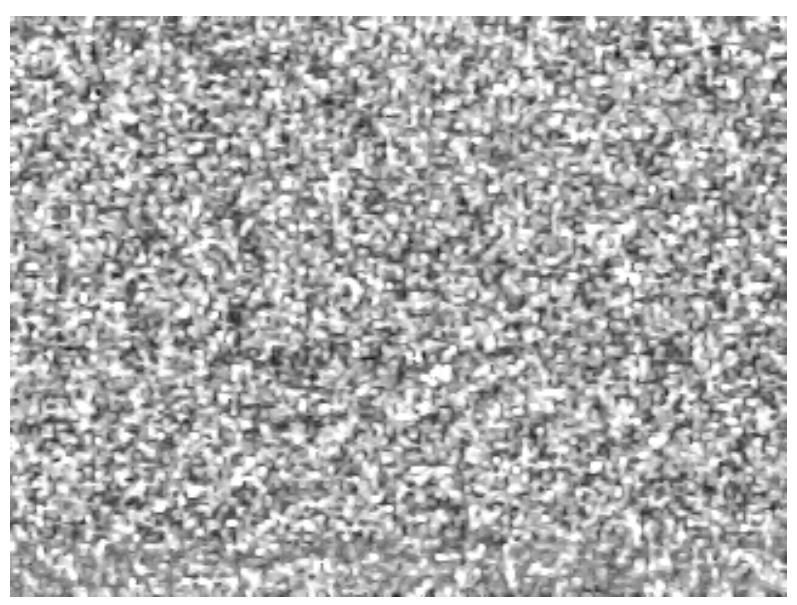

Figure 7. The obtained speckle pattern from partially coherent light.

coherent light.

By using software program (Image $J$ ), the value of the mean intensity $\langle I\rangle$ and the standard deviation $\sigma_{i}$ of the speckle pattern was obtained and substituted in the contrast equation.

$$
C=\sigma_{i} /\langle I\rangle
$$

Figure 8 shows the dependence of the speckle patterns contrast on the surface roughness obtained from the two spectral lines of the $\mathrm{Hg}$ lamp (the green and yellow line). To increase the range of spectral half width, we used the setup in Figure 9. As we can see from the figure, the collimated light illuminates a diffraction grating $D$. The spectrum of Halogen lamp appears beyond the diffraction grating. By using a rectangle aperture, we can select bands with different widths. For each band width we repeat the previous experimental procedures. Figure 10 shows the dependence of the speckle patterns contrast on the surface roughness for different spectral half widths of values $2.9 \times 10^{13} \mathrm{~Hz}, 2.59 \times 10^{13} \mathrm{~Hz}, 1.857 \times 10^{13} \mathrm{~Hz}$, $1.295 \times 10^{13} \mathrm{~Hz}$ respectively.

\section{Conclusion}

Effect of partially coherent light on the visibility was carried out. A theoretical model for a periodic rough sur- 


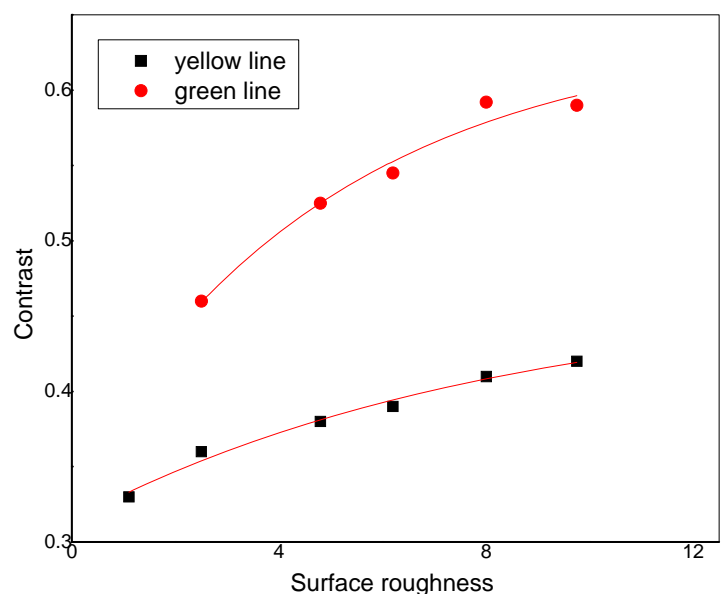

Figure 8. The relation between speckle patterns contrast and surface roughness.

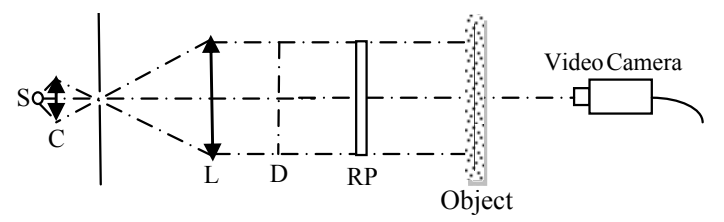

Figure 9. The experimental setup used to increase the range of the spectral half width.

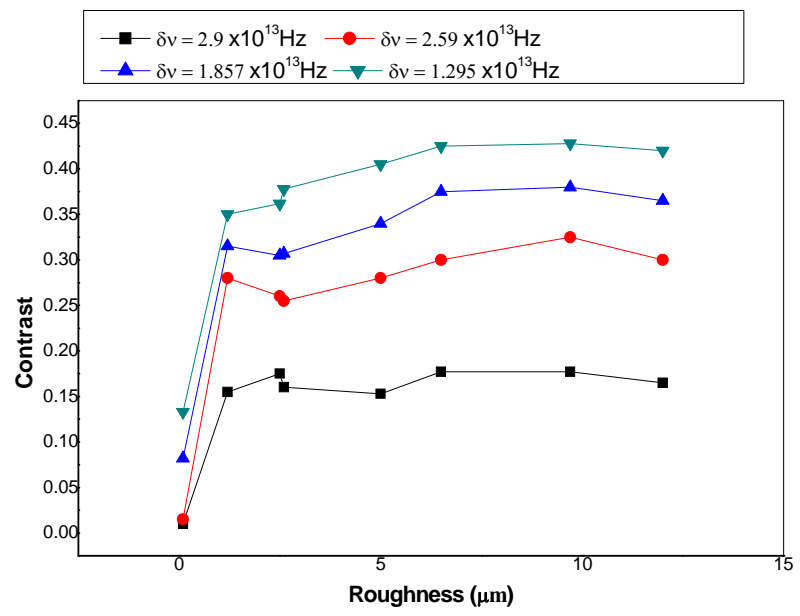

Figure 10. The relation between the speckle patterns contrast and surface roughness with $\Delta v=2.9 \times 10^{13} \mathrm{~Hz}, 2.59 \times$ $10^{13} \mathrm{~Hz}, 1.857 \times 10^{13} \mathrm{~Hz}$, and $1.295 \times 10^{13} \mathrm{~Hz}$ as a parameter.

face was constructed. Depending on the new theoretical model, an equation for the visibility of speckle patterns is investigated using partially coherent light. The general behavior of the experimental results, which agree with the published data, are in good agreement with the new theoretical model.

\section{REFERENCES}

[1] E. Archbuld, J. M. Burch and A. E. Ennos, "Recording of In-Plane Surface Displacement by Double-Exposure Speckle Photography," Optica Acta, Vol. 17, No. 12, 1970, pp. 883-898. doi: $10.1080 / 713818270$

[2] K. R. Erf, "Speckle Metrology," Chapter 4, Academic Press, New York, San Francisco, London, 1978.

[3] H. J. Tiziani, "Application of Speckling for In-Plane Vibration Analysis," Optica Acta, Vol. 18, No. 12, 1971, pp. 891-902. doi:10.1080/713818406

[4] H. Fujii and T. Asakura, "Coherence Measurement of Quasi Monochromatic Thermal Light Using Speckle Patterns 1," Optik, Vol. 39, 1973, pp. 99-117.

[5] H. Fujii and T. Asakura, "Coherence Measurement of Quasi Monochromatic Thermal Light Using Speckle Patterns II," Optik, Vol. 39, 1973, pp. 284-302.

[6] H. Fujji and T. Asakura, "A Contrast Variation of Image Speckle Intensity under Illumination of Partially Coherent Light," Optics Communications, Vol. 12, No. 1, 1974, pp. 32-38.

[7] T. Asakura, H. Fujii and K. Murata, "Measurement of Spatial Coherence Using Speckle Pattern," Optica Acta, Vol. 19, No. 4, 1972, pp. 273-290. doi:10.1080/713818561

[8] H. Fujiwara, T. Asakura and K. Murata, "Some Effects of Spatial and Temporal Coherence in Holography," Optica Acta, Vol. 17, No. 11, 1970, pp. 823-838. doi:10.1080/713818257

[9] C. Parry, "Some Effects of Temporal Coherence on the First Order Statistics of Speckle," Optica Acta, Vol. 21, No. 10, 1974, pp. 763-772. doi:10.1080/713818852

[10] E. A. Guillemin, "The Mathematics of Circuit Analysis," John Wiley and Sons, New York, 1951, p. 530.

[11] C. F. Cheng, C. X. Liu, N. Y. Zhang, T. Q. Jia, R. X. Li, and Z. Z. Xu, "Absolute Measurement of Roughness and Lateral-Correlation Length of Random Surfaces by Use of the Simplified Model of Image-Speckle Contrast," Applied Optics, Vol. 41, No. 20, 2002, pp. 4148-4156. doi:10.1364/AO.41.004148

[12] H. Fujji and T. Asakura, "Statistical Properties of Image Speckle Patterns in Partially Coherent Light," Nouvelle Revue d'Optique, Vol. 6, No. 1, 1975, pp. 5-14. doi:10.1088/0335-7368/6/1/301 